\title{
Gauge transformation and symmetries of the commutative multi-component BKP hierarchy
}

\author{
Chuanzhong Li \\ Department of Mathematics, Ningbo University, Ningbo, 315211, China \\ Email:lichuanzhong@nbu.edu.cn
}

\begin{abstract}
In this paper, we defined a new multi-component BKP hierarchy which takes values in a commutative subalgebra of $g l(N, \mathbb{C})$. After this, we give the gauge transformation of this commutative multi-component BKP (CMBKP) hierarchy. Meanwhile we construct a new constrained CMBKP hierarchy which contains some new integrable systems including coupled $\mathrm{KdV}$ equations under a certain reduction. After this, the quantum torus symmetry and quantum torus constraint on the tau function of the commutative multicomponent BKP hierarchy will be constructed.

Mathematics Subject Classifications(2000). 37K05, 37K10, 37K20.

Keywords: commutative multi-component BKP hierarchy, constrained commutative multicomponent BKP hierarchy, gauge transformation, quantum torus symmetry, quantum torus constraint.
\end{abstract}

\section{Contents}

1 Introduction 1

2 Lax equations of CMBKP hierarchy 3

3 Gauge transformations of the CMBKP hierarchy

4 Constrained CMBKP hierarchy 10

5 Quantum torus constraint of CMBKP hierarchy 11

6 Conclusions and Discussions

\section{Introduction}

The KP and Toda lattice hierarchies as completely integrable systems have many important applications in mathematics and physics including the theory of Lie algebras' 
representation, orthogonal polynomials and random matrix model [1] 6 . The KP hierarchy has many kinds of reduction or extension, for example the BKP, CKP hierarchies and so on. As important sub-hierarchies of the KP hierarchy, the constrained KP (cKP) hierarchy, the constrained BKP (cBKP) hierarchy and the constrained CKP(cCKP) hierarchy play an important role in the commutative integrable systems.

In [7, the Virasoro symmetry and ASvM formula of the BKP hierarchy were given. In 8, 9], the gauge transformations of the BKP, CKP, constrained BKP and constrained CKP hierarchies were constructed. In the paper [10], we construct the generalized additional symmetries of the two-component BKP hierarchy and identify its algebraic structure. As a reduction of the two-component BKP hierarchy, the D type Drinfeld-Sokolov hierarchy was found to be a good differential model to derive a complete Block type infinite dimensional Lie algebra (also called Torus Lie algebra). About the Block algebra and its quantization (quantum torus algebra) related to integrable systems, we did a series of works in [11][14. In the paper [15], we constructed the additional symmetries of the supersymmetric BKP hierarchy which constitute a B type $S W_{1+\infty}$ Lie algebra. Further we generalize the SBKP hierarchy to a supersymmetric two-component BKP hierarchy (S2BKP) hierarchy and a new supersymmetric Drinfeld-Sokolov hierarchy of type D which has a super Block type additional symmetry.

There is another kind of generalization of KP and Toda systems called multi-component KP [16, 17] or multi-component Toda system which attracts more and more attention because of its widely use in many fields such as the fields of multiple orthogonal polynomials and non-intersecting Brownian motions. In [18, they considered a generalized multicomponent KP hierarchy which contains $\mathrm{N}$ independent generalized scalar KP hierarchies in particular by considering a commutative subalgebra of diagonal matrices. In [19], a formalism of multicomponent BKP hierarchies using elementary geometry of spinors was developed by Kac and van de Leur. In [20, M. Mañas, Luis Martínez Alonso construct a relation between multicomponent BKP hierarchy and Lame equations from Ramond fermions. The $\tau$ functions of a $2 N$-multicomponent KP hierarchy provide solutions of the $N$-multicomponent two dimensional Toda hierarchy [4] which was considered from the point of view of the theory of multiple matrix orthogonal polynomials, non-intersecting Brownian motions and matrix Riemann-Hilber problem [21]- 22]. The multicomponent Toda hierarchy in [21] is a periodic reduction of bi-infinite matrix-formed Toda hierarchy which contains matrix-formed Toda equation as the first flow equation. In [23], we defined the extended multi-component Toda hierarchy and its Sato theory.

In [24], a new hierarchy called as $Z_{m}$-KP hierarchy which take values in a maximal commutative subalgebra of $g l(m, \mathbb{C})$ was constructed, meanwhile the relation between Frobenius manifold and dispersionless reduced $Z_{m}$-KP hierarchy was discussed. This inspired us to consider the Hirota quadratic equation of the commutative version of extended multi-component Toda hierarchy in [25] which might be useful in Frobenius manifold theory.

This paper is arranged as follows. In the next section we recall the factorization problem and construct the multicomponent $Z_{N}$-BKP hierarchy. In Section 2, we will give the Lax equations of the commutative multicomponent BKP hierarchy. In Section 3, multi-fold transformations of the CMBKP hierarchy will be constructed using the determinant technique in [26, 27]. We construct a new constrained CMBKP hierarchy which contains some new integrable systems including a coupled commutative matrix $K d V$ equation in Section 4. In Section 5, the quantum torus symmetry and quantum torus constraint on the tau function of the commutative multi-component BKP hierarchy 
will be constructed. Section 6 will be devoted to a short conclusions and discussions.

\section{Lax equations of CMBKP hierarchy}

In this section we will use the factorization problem to derive Lax equations. We will consider the linear space of the complex $N \times N$ matrix-valued function $g: \mathbb{R} \rightarrow M_{N}(\mathbb{C})$ with the derivative operator $\partial$. Then the set $\mathfrak{g}$ of Laurent series in $\partial$ as an associative algebra is a Lie algebra under the standard commutator. This Lie algebra has the following important splitting

$$
\mathfrak{g}=\mathfrak{g}_{+} \oplus \mathfrak{g}_{-},
$$

where

$$
\mathfrak{g}_{+}=\left\{\sum_{j \geq 0} X_{j}(x) \partial^{j}, \quad X_{j}(x) \in M_{N}(\mathbb{C})\right\}, \quad \mathfrak{g}_{-}=\left\{\sum_{j<0} X_{j}(x) \partial^{j}, \quad X_{j}(x) \in M_{N}(\mathbb{C})\right\} .
$$

The splitting (11) leads us to consider the following factorization of $g \in G$

$$
g=g_{-}^{-1} \circ g_{+}, \quad g_{ \pm} \in G_{ \pm}
$$

where $G_{ \pm}$have $\mathfrak{g}_{ \pm}$as their Lie algebras. $G_{+}$is the set of invertible linear operators of the form $\sum_{j \geq 0} g_{j}(x) \partial^{j}$; while $G_{-}$is the set of invertible linear operators of the form $1+\sum_{j<0} g_{j}(x) \partial^{j}$. This algebra has a maximal commutative subalgebra $Z_{N}=\mathbb{C}[\Gamma] /\left(\Gamma^{N}\right)$ and $\Gamma=\left(\delta_{i, j+1}\right)_{i j} \in g l(N, \mathbb{C})$. Denote $Z_{N}(\partial):=\mathfrak{g}_{c}$, then we have the following splitting

$$
\mathfrak{g}_{c}=\mathfrak{g}_{c+} \oplus \mathfrak{g}_{c-},
$$

where

$$
\mathfrak{g}_{c+}=\left\{\sum_{j \geq 0} X_{j}(x) \partial^{j}, \quad X_{j}(x) \in Z_{N}\right\}, \quad \mathfrak{g}_{c-}=\left\{\sum_{j<0} X_{j}(x) \partial^{j}, \quad X_{j}(x) \in Z_{N}\right\} .
$$

We denote "*" as a formal adjoint operation defined by $p^{*}=\sum(-1)^{i} \partial^{i} \circ p_{i}$ for an arbitrary $Z_{N}$-valued pseudo-differential operator $p=\sum p_{i} \partial^{i}$, and $(f g)^{*}=g^{*} f^{*}$ for two operators $(f, g)$. Here $\circ$ means the multiplication of two operators.

Before the work, we list some identities, which will be used in the following sections:

$$
\begin{gathered}
A^{*}=A, \\
(A B)^{*}=B A, \\
(A \circ \partial \circ B)^{*}=-B \circ \partial \circ A,
\end{gathered}
$$

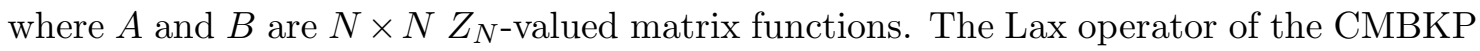
hierarchy has form

$$
L=\partial+\sum_{i \geq 1} u_{i} \partial^{-i}
$$


where $u_{i}$ takes values in the commutative subalgebra $Z_{N}$. And the operator must satisfy the following so-called B type condition

$$
L^{*}=-\partial \circ L \circ \partial^{-1}
$$

The CMBKP hierarchy is defined by the following Lax equations:

$$
\partial_{2 k-1} L=\left[\left(B_{2 k-1}\right)_{+}, L\right], \quad B_{2 k-1}=L^{2 k-1}, \quad k \geq 1 .
$$

One can write the operators $L$ in a dressing form as

$$
L=\Phi \circ \partial \circ \Phi^{-1}
$$

where

$$
\Phi=1+\sum_{i \geq 1} a_{i} \partial^{-i}
$$

satisfy

$$
\Phi^{*}=\partial \circ \Phi^{-1} \circ \partial^{-1} .
$$

We call eq.(12) the B type condition of the CMBKP hierarchy. Given $L$, the dressing operators $\Phi$ are determined uniquely up to a multiplication to the right by operators with constant coefficients. The dressing operator $\Phi$ takes values in a B type commutative Volterra group in $G_{-}$. The CMBKP hierarchy (23) can also be redefined as

$$
\frac{\partial \Phi}{\partial t_{2 k-1}}=-\left(L^{2 k-1}\right)_{-} \circ \Phi
$$

with $k \geq 1$. In the the CMBKP hierarchy, we can derive an equation as following

$$
9 v_{x, t_{5}}-5 v_{t_{3}, t_{3}}+\left(v_{x x x x x}-5 v_{x x, t_{3}}-15 v_{x} v_{t_{3}}+15 v_{x} v_{x x x}+15 v_{x}^{3}\right)_{x}=0
$$

where $v=\int u_{1} d x$ is in the $Z_{N}$ algebra. We will call the eq.114 the CMBKP equation. When $N=2$, we can derive the following two-component CMBKP equation as

$9 w_{x, t_{5}}-5 w_{t_{3}, t_{3}}+\left(w_{x x x x x}-5 w_{x x, t_{3}}-15 w_{x} w_{t_{3}}+15 w_{x} w_{x x x}+15 w_{x}^{3}\right)_{x}=0,(15)$

$9 z_{x, t_{5}}-5 z_{t_{3}, t_{3}}+\left(z_{x x x x x}-5 z_{x x, t_{3}}-15 w_{x} z_{t_{3}}-15 z_{x} w_{t_{3}}+15 w_{x} z_{x x x}+15 z_{x} w_{x x x}+45 z_{x} w_{x}^{2}\right)_{x}=0,(16)$

where $v=w+z \Gamma$. After freezing the $t_{3}$ flow, the CMBKP equation will be reduced to commutative two-component Sawada-Kotera(CMSK) equation as

$$
\begin{array}{r}
9 w_{x, t_{5}}+\left(w_{x x x x x}+15 w_{x} w_{x x x}+15 w_{x}^{3}\right)_{x}=0 \\
9 z_{x, t_{5}}+\left(z_{x x x x x}+15 w_{x} z_{x x x}+15 z_{x} w_{x x x}+45 z_{x} w_{x}^{2}\right)_{x}=0 .
\end{array}
$$

With the above preparation, it is time to construct gauge transformations for the CMBKP hierarchy in the next section. 


\section{Gauge transformations of the CMBKP hierar- chy}

In this section, we will consider the gauge transformation of the CMBKP hierarchy on the Lax operator

$$
L^{[1]}=\partial+\sum_{i \geq 1} U_{i}^{[1]} \partial^{-i}=W \circ L \circ W^{-1}
$$

where $W$ is the gauge transformation operator. And $L^{[1]}$ should satisfy the B type condition

$$
\left(L^{[1]}\right)^{*}=-\partial \circ L^{[1]} \circ \partial^{-1},
$$

which further implies

$$
W^{*}=\partial \circ W^{-1} \circ \partial^{-1}
$$

That means after the gauge transformation, the spectral problem about the $N \times N$ spectral matrix $\phi$ taking values in the commutative subalgebra $Z_{N}$ will preserve its form as

$$
L \cdot \phi=\lambda \phi, \quad \frac{\partial \phi}{\partial t_{n}}=B_{n} \cdot \phi .
$$

To keep the Lax pair of the CMBKP hierarchy invariant, i.e.,

$$
\partial_{t_{n}} L^{[1]}=\left[\left(B_{n}^{[1]}\right)_{+}, L^{[1]}\right], \quad B_{n}^{[1]}=\left(L^{[1]}\right)^{n}, \quad n=1,3,5, \cdots,
$$

the dressing operator $W$ should satisfy the following dressing equation

$$
W_{t_{n}}=-W \circ\left(B_{n}\right)_{+}+\left(W \circ B_{n} \circ W^{-1}\right)_{+} \circ W, \quad n=1,3,5 \cdots,
$$

where $W_{t_{n}}$ means the derivative of $W$ by $t_{n}$.

The evolutions of the eigenfunction $\phi$ and the adjoint eigenfunction $\psi$ of the CMBKP hierarchy are defined respectively by

$$
\frac{\partial \phi}{\partial t_{n}}=B_{n} \cdot \phi, \frac{\partial \psi}{\partial t_{n}}=-\left(B_{n}\right)^{*} \cdot \psi
$$

where $\phi=\phi(\lambda ; t)$ and $\psi=\psi(\lambda ; t)$ and $t=\left(t_{1}, t_{3}, t_{5}, \ldots\right)$. To give the gauge transformation, we need the following lemma.

Lemma 1. The operator $B:=\sum_{n=0}^{\infty} b_{n} \partial^{n}\left(B:=\sum_{n=0}^{\infty} \partial^{n} \circ a_{n}\right)$ is a $Z_{N}$-valued differential operator and $f, g$ (short for $f(x), g(x)$ ) are two matrix functions taking values in the commutative subalgebra $Z_{N}$, following identities hold

$$
\left(B \circ f \partial^{-1} \circ g\right)_{-}=(B \cdot f) \circ \partial^{-1} \circ g, \quad\left(f \partial^{-1} \circ g \circ B\right)_{-}=f \partial^{-1} \circ\left(B^{*} \cdot g\right) .
$$


Proof. Here we only give the proof of the second equation of (26) by direct calculation basing on the first equation of (26)

$$
\begin{aligned}
\left(f \partial^{-1} \circ g \circ B\right)_{-} & =\left(-B^{*} \circ g \circ \partial^{-1} \circ f\right)_{-}^{*} \\
& =\left(\left(-B^{*} \cdot g\right) \circ \partial^{-1} \circ f\right)^{*} \\
& =\sum_{m=0}^{\infty}\left(-a_{m}\left((-\partial)^{m} \cdot g\right) \partial^{-1} \circ f\right)^{*} \\
& =\sum_{m=0}^{\infty}(-1)^{m} f \partial^{-1} \circ\left(\partial^{m} \cdot g\right)^{*} \circ a_{m} \\
& =\sum_{m=0}^{\infty} f \partial^{-1}(-1)^{m} \circ a_{m}\left(\partial^{m} \cdot g\right) \\
& =f \partial^{-1} \circ\left(B^{*} \cdot g\right) .
\end{aligned}
$$

Lemma 2. The operators $T_{D}=\phi \circ \partial \circ \phi^{-1}$ and $T_{I}=\psi^{-1} \circ \partial^{-1} \circ \psi$ satisfy eq.(24) , which implies $T_{D} T_{I}=\phi \circ \partial \circ \phi^{-1} \circ \psi^{-1} \circ \partial^{-1} \circ \psi$ can also satisfy eq.(24).

Now, we will find out the gauge transformation operator $W$ of the CMBKP hierarchy. Firstly, we consider the two operators

$$
T_{D}(\phi)=\phi \circ \partial \circ \phi^{-1}, T_{I}(\psi)=\psi^{-1} \circ \partial^{-1} \circ \psi,
$$

where $\phi$ and $\psi$ are $N \times N$ matrix-valued eigenfunctions taking values in the commutative subalgebra $Z_{N}$. Then we have

$$
\left(T_{D}^{-1}(\phi)\right)^{*}=-T_{I}(\phi),\left(T_{I}^{-1}(\psi)\right)^{*}=-T_{D}(\psi) .
$$

We can easily get

$$
T_{D}(\phi) \cdot \phi=0,\left(T_{I}^{-1}(\psi)\right)^{*} \cdot \psi=0
$$

Similarly to the reference [26], we can consider two sets of matrix functions $\left\{\phi_{i}^{(0)}, i=1,2, \ldots n ; \phi^{(0)}\right\}$ and $\left\{\psi_{i}^{(0)}, i=1,2 \cdots n ; \psi^{(0)}\right\}$. For $T_{D}(\phi)=\phi \circ \partial \circ \phi^{-1}$, we do iterations by the following two steps. For the first step, we consider:

$$
T_{D}^{(1)}=T_{D}^{(1)}\left(\phi_{1}^{(0)}\right)=\phi_{1}^{(0)} \circ \partial \circ\left(\phi_{1}^{(0)}\right)^{-1},
$$

we define the rule of transformation under $T_{D}^{(1)}$ as

$$
\begin{aligned}
& \phi^{(1)}=T_{D}^{(1)}\left(\phi_{1}^{(0)}\right) \cdot \phi^{(0)}, \psi^{(1)}=\left(T_{D}^{(1)}\left(\phi_{1}^{(0)}\right)\right)^{*^{-1}} \cdot \psi^{(0)}=-T_{I}\left(\phi_{1}^{(0)}\right) \cdot \psi^{(0)}, \\
& \phi_{i}^{(1)}=T_{D}^{(1)}\left(\phi_{1}^{(0)}\right) \cdot \phi_{i}^{(0)}, \psi_{i}^{(1)}=\left(T_{D}^{(1)}\left(\phi_{1}^{(0)}\right)\right)^{*^{-1}} \cdot \psi_{i}^{(0)}=-T_{I}\left(\phi_{1}^{(0)}\right) \cdot \psi_{i}^{(0)},
\end{aligned}
$$

where $i \geqslant 2$ for $\phi_{i}^{(1)}$ and

$$
\psi_{i}^{(1)}=-T_{I}\left(\phi_{1}^{(0)}\right) \cdot\left(\psi_{i}^{(0)}\right)
$$


For the second step, we consider:

$$
T_{D}^{(2)}=T_{D}^{(2)}\left(\phi_{2}^{(1)}\right)=\phi_{2}^{(1)} \circ \partial \circ\left(\phi_{2}^{(1)}\right)^{-1},
$$

we define the rule of transformation under $T_{D}^{(2)}$ as

$$
\begin{aligned}
& \phi^{(2)}=T_{D}^{(2)}\left(\phi_{2}^{(1)}\right) \cdot \phi^{(1)}, \psi^{(2)}=\left(T_{D}^{(2)}\left(\phi_{2}^{(1)}\right)\right)^{*^{-1}} \cdot \psi^{(1)}=-T_{I}\left(\left(\phi_{2}^{(1)}\right)\right) \cdot \psi^{(1)}, \\
& \phi_{i}^{(2)}=T_{D}^{(2)}\left(\phi_{2}^{(1)}\right) \cdot \phi_{i}^{(1)}, \psi_{i}^{(2)}=\left(T_{D}^{(2)}\left(\phi_{2}^{(1)}\right)\right)^{*^{-1}} \cdot \psi_{i}^{(1)}=-T_{I}\left(\left(\phi_{2}^{(1)}\right)\right) \cdot \psi_{i}^{(1)},
\end{aligned}
$$

where $i \geqslant 3$ for $\phi_{i}^{(2)}$ and

$$
\psi_{i}^{(2)}=-T_{I}\left(\left(\phi_{2}^{(1)}\right)\right) \cdot\left(\psi_{i}^{(1)}\right) .
$$

For $T_{I}(\psi)=\psi^{-1} \circ \partial^{-1} \circ \psi$, it obeys the following iterated rule:

For the first step, we consider:

$$
\begin{gathered}
T_{I}^{(1)}=T_{I}^{(1)}\left(\psi_{1}^{(0)}\right)=\left(\psi_{1}^{(0)}\right)^{-1} \circ \partial^{-1} \circ\left(\psi_{1}^{(0)}\right), \\
\phi^{(1)}=T_{I}^{(1)}\left(\psi_{1}^{(0)}\right) \cdot \phi^{(0)}, \psi^{(1)}=\left(T_{I}^{(1)}\left(\psi_{1}^{(0)}\right)\right)^{*^{-1}} \cdot \psi^{(0)}=-T_{D}\left(\left(\psi_{1}^{(0)}\right)\right) \cdot \psi^{(0)}, \\
\phi_{i}^{(1)}=T_{I}^{(1)}\left(\psi_{1}^{(0)}\right) \cdot \phi_{i}^{(0)}, \psi_{i}^{(1)}=\left(T_{I}^{(1)}\left(\psi_{1}^{(0)}\right)\right)^{*^{-1}} \cdot \psi_{i}^{(0)}=-T_{D}\left(\left(\psi_{1}^{(0)}\right)\right) \cdot \psi_{i}^{(0)},
\end{gathered}
$$

where $i \geqslant 2$ for $\psi_{i}^{(1)}$ and

$$
\psi_{i}^{(1)}=-T_{D}\left(\left(\psi_{1}^{(0)}\right)\right) \cdot\left(\psi_{i}^{(0)}\right)
$$

For the second step, we consider:

$$
\begin{gathered}
T_{I}^{(2)}=T_{I}^{(2)}\left(\psi_{2}^{(1)}\right)=\left(\psi_{2}^{(1)}\right)^{-1} \circ \partial^{-1} \circ\left(\psi_{2}^{(1)}\right), \\
\phi^{(2)}=T_{I}^{(2)}\left(\psi_{2}^{(1)}\right) \cdot \phi^{(1)}, \psi^{(2)}=\left(T_{I}^{(2)}\left(\psi_{2}^{(1)}\right)\right)^{*^{-1}} \cdot \psi^{(1)}=-T_{D}\left(\psi_{2}^{(1)}\right) \cdot \psi^{(1)}, \\
\phi_{i}^{(2)}=T_{I}^{(2)}\left(\psi_{2}^{(1)}\right) \cdot \phi_{i}^{(1)}, \psi_{i}^{(2)}=\left(T_{I}^{(2)}\left(\psi_{2}^{(1)}\right)\right)^{*^{-1}} \cdot \psi_{i}^{(1)}=-T_{D}\left(\psi_{2}^{(1)}\right) \cdot \psi_{i}^{(1)},
\end{gathered}
$$

where $i \geqslant 3$ for $\psi_{i}^{(1)}$ and

$$
\psi_{i}^{(2)}=-T_{D}\left(\psi_{2}^{(1)}\right) \cdot\left(\psi_{i}^{(1)}\right) .
$$

It is obvious that a single step of the operator $T_{D}$ or $I_{I}$ can not keep the restriction of the B type condition, we use

$$
W_{1}=T_{1+1}=T_{I}\left(\psi_{1}^{(1)}\right) \circ T_{D}\left(\phi_{1}^{(0)}\right),
$$


as the gauge transformation operator and we have $L^{[1]}=W_{1} L W_{1}^{-1}$. Let us check whether it satisfies the required constraint

$$
\left(L^{[1]}\right)^{*}=-\partial L^{[1]} \partial^{-1},
$$

We can calculate

$$
\begin{aligned}
\left(L^{[1]}\right)^{*}= & \left(\left(\psi_{1}^{(1)}\right)^{-1} \circ \partial^{-1} \circ \psi_{1}^{(1)} \circ \phi_{1}^{(0)} \circ \partial \circ\left(\phi_{1}^{(0)}\right)^{-1} \circ\right. \\
& \left.L \circ \phi_{1}^{(0)} \circ \partial^{-1} \circ\left(\phi_{1}^{(0)}\right)^{-1} \circ\left(\psi_{1}^{(1)}\right)^{-1} \circ \partial \circ \psi_{1}^{(1)}\right)^{*} \\
= & -\left(\psi_{1}^{(1)}\right) \circ \partial \circ\left(\left(\psi_{1}^{(1)}\right)\right)^{-1}\left(\phi_{1}^{(0)}\right)^{-1} \circ \partial^{-1} \circ\left(\phi_{1}^{(0)}\right) \circ \partial \circ L \\
& \circ \partial^{-1} \circ\left(\phi_{1}^{(0)}\right)^{-1} \circ \partial \circ\left(\phi_{1}^{(0)}\right) \circ\left(\psi_{1}^{(1)}\right) \circ \partial^{-1} \circ\left(\left(\psi_{1}^{(1)}\right)\right)^{-1},
\end{aligned}
$$

and

$$
\begin{array}{r}
-\partial L^{[1]} \partial^{-1}=-\partial \circ\left(\left(\psi_{1}^{(1)}\right)\right)^{-1} \circ \partial^{-1} \circ \psi_{1}^{(1)} \circ \phi_{1}^{(0)} \circ \partial \circ\left(\phi_{1}^{(0)}\right)^{-1} \\
\circ L \circ \phi_{1}^{(0)} \circ \partial^{-1} \circ\left(\phi_{1}^{(0)}\right)^{-1} \circ\left(\psi_{1}^{(1)}\right)^{-1} \circ \partial \circ \psi_{1}^{(1)} \circ \partial^{-1},
\end{array}
$$

which means in order to keep the constraint $\left(L^{[1]}\right)^{*}=-\partial L^{[1]} \partial^{-1}, T$ should satisfy the following equation:

$$
T_{D}\left(\psi_{1}^{(1)}\right) T_{I}\left(\phi_{1}^{(0)}\right) \circ \partial=\partial \circ T_{I}\left(\psi_{1}^{(1)}\right) T_{D}\left(\phi_{1}^{(0)}\right),
$$

where $\psi_{1}^{(1)}=-\left(\phi_{1}^{(0)}\right)^{-1} \int\left(\phi_{1}^{(0)}\right) \psi_{1}^{(0)}$ and $\int$ means the integral about spatial variable $x$.

Then we can acquire the following theorem because the CMBKP hierarchy takes values in a commutative subalgebra just like the case when $N=1$, i.e. the case of the original BKP hierarchy..

Theorem 1. The B type condition of the CMBKP hierarchy implies $\psi_{1}^{(0)}$ and $\phi_{1}^{(0)}$ have the following relation:

$$
\psi_{1}^{(0)}=\phi_{1, x}^{(0)}
$$

The B-type reduction of $L$ guarantee that there exists at least one solution $(\phi ; \psi)$ which satisfies eq.(52). In fact the above theorem can be generalized to the case of the $g l(N, \mathbb{C})$-valued multicomponent BKP hierarchy which is not commutative.

The B type condition of the $g l(N, \mathbb{C})$-valued multicomponent BKP hierarchy implies noncommutative matrices $\psi_{1}^{(0)}$ and $\phi_{1}^{(0)}$ have the following relation:

$\left(\left(\phi_{1}^{(0)}\right)^{T}\right)^{-2}\left(\phi_{1}^{(0)}\right)_{x}^{T} \int\left(\phi_{1}^{(0)}\right)^{T} \psi_{1}^{(0)}-\psi_{1}^{(0)}-\left(\left(\phi_{1}^{(0)}\right)^{T}\right)^{-1}\left(\int\left(\phi_{1}^{(0)}\right)^{T} \psi_{1}^{(0)}\right) \phi_{1, x}^{(0)}\left(\phi_{1}^{(0)}\right)^{-1}+\left(\phi_{1}^{(0)}\right)_{x}^{T}=0$

where $T$ means the transpose of matrices.

The proof of the eq.(53) will be skipped here because the focus of this paper is about the CMBKP hierarchy. A thorough study on the $g l(N, \mathbb{C})$-valued multicomponent BKP hierarchy will be contained in another work of ours recently.

Remark: From eq.(52) to eq.(53), one can see clearly the difference of the BKP systems from $Z_{N}$ to $g l(N, \mathbb{C})$.

In order to keep the B type restriction of the Lax operator of the CMBKP hierarchy, we do iterations of the gauge transformation $W_{n}=T_{n+n}$. In particular,

$$
W_{2}=T_{2+2}=T_{I}\left(\psi_{2}^{(3)}\right) \circ T_{D}\left(\phi_{2}^{(2)}\right) \circ T_{I}\left(\psi_{1}^{(1)}\right) \circ T_{D}\left(\phi_{1}^{(0)}\right),
$$




$$
W_{n}=T_{n+n}=T_{I}\left(\psi_{n}^{(2 n-1)}\right) \circ T_{D}\left(\phi_{n}^{(2 n-2)}\right) \circ \ldots \circ T_{I}\left(\psi_{1}^{(1)}\right) \circ T_{D}\left(\phi_{1}^{(0)}\right),
$$

where $\psi_{i}^{(2 n-1)}=-T_{I}\left(\left(\phi_{n}^{(2 n-2)}\right)\right) \cdot\left(\psi_{i}^{(2 n-2)}\right), \quad \psi_{n}^{(i)}=\left(\phi_{n}^{(i)}\right)_{x}$. It can be easily checked that $\left.W_{n} \cdot \phi_{i}^{(0)}\right|_{i \leq n}=0,\left.\left(W_{n}^{-1}\right)^{*} \cdot\left(\psi_{i}^{(0)}\right)\right|_{i \leq n}=0$. The relations $\psi_{n}^{(i)}=\left(\phi_{n}^{(i)}\right)_{x}, \quad n=1,2 \ldots$ can keep the dressing procedures $W_{n}=T_{n+n}, \quad n=1,2 \ldots$ always preserving the $\mathrm{B}$ type condition of new Lax operators $L^{[n]}$. This is similar as the case of the BKP hierarchy in [8].

We denote $t=\left(t_{1}, t_{3}, t_{5}, \ldots\right)$ and introduce the $Z_{N}$-valued wave function as

$$
w(t ; z)=\Phi \cdot e^{\xi(t ; z)},
$$

where the function $\xi$ is defined as $\xi(t ; z)=\sum_{k \in \mathbb{Z}_{+}^{\text {odd }}} t_{k} z^{k}$. It is easy to see

$$
L w(t ; z)=z w(t ; z), \quad \frac{\partial w}{\partial t_{2 n+1}}=L_{+}^{2 n+1} w .
$$

The $Z_{N}$-valued tau function $\tau$ of the CMBKP hierarchy can be defined in form of the wave functions as

$$
w(t, z)=\frac{\tau\left(t-2\left[z^{-1}\right]\right)}{\tau(t)} e^{\xi(t ; z)}
$$

where $[z]=\left(z, z^{3} / 3, z^{5} / 5, \ldots\right)$.

The generating functions of n-step $T_{D}$ and n-step $T_{I}$ are denoted as $\left(\phi_{1}, \ldots, \phi_{n-1}, \phi_{n}\right)$ and $\left(\psi_{1}, \ldots, \psi_{n-1}, \psi_{n}\right)$ in order respectively. The generating functions have the following B type constraint

$$
\psi_{i}=\left(\phi_{i}\right)_{x}
$$

Using the above gauge transformation, we can derive the gauge transformation on the tau function of the CMBKP hierarchy as

$$
\tau^{(n+n)}=G W_{n, n}\left(\psi_{n}, \psi_{n-1}, \ldots, \psi_{1} ; \phi_{1}, \ldots, \phi_{n-1}, \phi_{n}\right) \tau,
$$

where the generalized Wronskian $G W_{k, n}$ is defined as 9 ]

$$
\begin{aligned}
& G W_{k, n}\left(g_{k}, g_{k-1}, \ldots, g_{1} ; f_{1}, f_{2}, \ldots, f_{n}\right) \\
& =\left|\begin{array}{ccccc}
\int g_{k} f_{1} & \int g_{k} f_{2} & \int g_{k} f_{3} & \ldots & \int g_{k} f_{n} \\
\int g_{k-1} f_{1} & \int g_{k-1} f_{2} & \int g_{k-1} f_{3} & \ldots & \int g_{k-1} f_{n} \\
\vdots & \vdots & \vdots & \ldots & \vdots \\
\int g_{1} f_{1} & \int g_{1} f_{2} & \int g_{1} f_{3} & \ldots & \int g_{1} f_{n} \\
f_{1} & f_{2} & f_{3} & \ldots & f_{n} \\
f_{1 x} & f_{2 x} & f_{3 x} & \ldots & f_{n x} \\
\vdots & \vdots & \vdots & \ldots & \vdots \\
\left(f_{1}\right)^{(n-k-1)} & \left(f_{2}\right)^{(n-k-1)} & \left(f_{3}\right)^{(n-k-1)} & \ldots & \left(f_{n}\right)^{(n-k-1)}
\end{array}\right| .
\end{aligned}
$$

When $k=0$, the generalized Wronskian $G W_{0, n}$ will be reduced to the ordinary Wronskian. Now, we will only give the first gauge transformation of the CMBKP hierarchy included in the following proposition.

Proposition 1. If the eigenfunction $\phi$ and the adjoint eigenfunction $\psi$ satisfy the eq.(25), the one-fold gauge transformation operator of the CMBKP hierarchy

$$
W_{1}=\left(\psi_{1}^{(1)}\right)^{-1} \circ \partial^{-1} \circ \psi_{1}^{(1)} \circ \phi_{1}^{(0)} \circ \partial \circ\left(\phi_{1}^{(0)}\right)^{-1},
$$


satisfies $W_{1} \phi_{1}^{(0)}=0$ and $\left(W_{1}^{-1}\right)^{*}\left(\psi_{1}^{(0)}\right)=0 . W_{1}$ will generater new solutions $U_{i}^{[1]}$ from seed solutions $U_{i}$. To see it clearly, here we only give the transformations of first two dynamic functions

$$
\begin{gathered}
U_{1}^{[1]}=U_{1}+2\left(\ln \phi_{1}^{(0)}\right)_{x x} \\
U_{2}^{[1]}=U_{2}+4\left(\ln \phi_{1}^{(0)}\right)_{x x}\left(\ln \phi_{1}^{(0)}\right)_{x}-2\left(\frac{\psi_{1, x}^{(0)}}{\phi_{1}^{(0)}}\right)_{x} .
\end{gathered}
$$

If we suppose $U_{1}=\left[\begin{array}{cc}\alpha & 0 \\ \beta & \alpha\end{array}\right], U_{2}=\left[\begin{array}{ll}\gamma & 0 \\ \eta & \gamma\end{array}\right], \phi_{1}^{(0)}=\left[\begin{array}{cc}\phi_{0} & 0 \\ \phi_{1} & \phi_{0}\end{array}\right]$, then we can derive the explicit transformation as

$$
\begin{aligned}
& \alpha^{[1]}=\alpha+2\left(\ln \phi_{0}\right)_{x x}, \\
& \beta^{[1]}=\beta+2\left(\frac{\phi_{1}}{\phi_{0}}\right)_{x x} \\
& \gamma^{[1]}=\gamma+4\left(\ln \phi_{0}\right)_{x x}\left(\ln \phi_{0}\right)_{x}-2\left(\frac{\phi_{0 x x}}{\phi_{0}}\right)_{x}, \\
& \eta^{[1]}=\eta+4\left(\ln \phi_{0}\right)_{x x}\left(\frac{\phi_{1}}{\phi_{0}}\right)_{x}+4\left(\frac{\phi_{1}}{\phi_{0}}\right)_{x x}\left(\ln \phi_{0}\right)_{x}-2\left(\frac{\phi_{1 x x}}{\phi_{0}}-\frac{\phi_{0 x x} \phi_{1}}{\phi_{0}^{2}}\right)_{x} .
\end{aligned}
$$

In the calculation, the identity $\ln \left[\begin{array}{cc}\phi_{0} & 0 \\ \phi_{1} & \phi_{0}\end{array}\right]=\left[\begin{array}{cc}\ln \phi_{0} & 0 \\ \frac{\phi_{1}}{\phi_{0}} & \ln \phi_{0}\end{array}\right]$ is used.

For the case $N=1, W_{1}$ will generater new solutions of the BKP hierarchy from seed solutions.

\section{Constrained CMBKP hierarchy}

In this section, we will consider the operator of the constrained CMBKP(cCMBKP) hierarchy as

$$
L=\partial+u \partial^{-1} v_{x}-v \partial^{-1} u_{x}
$$

where $u$ and $v$ are $N \times N$ matrix functions taking values in $Z_{N}$. Here $u, v$ should satisfy the following Sato equation

$$
u_{t_{2 n-1}}=B_{2 n-1} \cdot u, v_{t_{2 n-1}}=B_{2 n-1} \cdot v .
$$

Because the B type condition eq. (8), one can prove that the $\partial^{0}$ term does not exist in $B_{2 n-1}$ as mentioned in [1]. That means that $u=v=1$ is a trivial solution.

Suppose $u=q+p \Gamma, v=r+s \Gamma$, and consider the case when $N=2$, i.e.

$$
u=\left[\begin{array}{ll}
q & 0 \\
p & q
\end{array}\right], \quad v=\left[\begin{array}{ll}
r & 0 \\
s & r
\end{array}\right] .
$$

Then we can further derive the following coupled equations

$$
\begin{aligned}
q_{t_{3}} & =q_{3 x}+3\left(q r_{x}-r q_{x}\right) q_{x} \\
p_{t_{3}} & =p_{3 x}+3\left[\left(q r_{x}-r q_{x}\right) p_{x}+\left(p r_{x}+q s_{x}-s q_{x}-r p_{x}\right) q_{x}\right] \\
r_{t_{3}} & =r_{3 x}+3\left(q r_{x}-r q_{x}\right) r_{x} \\
s_{t_{3}} & =s_{3 x}+3\left[\left(q r_{x}-r q_{x}\right) s_{x}+\left(p r_{x}+q s_{x}-s q_{x}-r p_{x}\right) r_{x}\right] .
\end{aligned}
$$


If $q=r, p=s$, we can derive the following trivial equations

$$
\begin{aligned}
& q_{t_{3}}=q_{3 x}, \\
& p_{t_{3}}=p_{3 x} .
\end{aligned}
$$

If $r=s=1$, we can derive the following coupled matrix KdV-like equation

$$
\begin{aligned}
& q_{t_{3}}=q_{3 x}-3 q_{x}^{2}, \\
& p_{t_{3}}=p_{3 x}-6 q_{x} p_{x}-3 q_{x}^{2} .
\end{aligned}
$$

Similarly as [9], we can derive the new solutions generated from the seed solution $q, r$

$$
\begin{gathered}
u^{(n+n)}=\frac{G W_{n, n+1}\left(\psi^{(n-1)}, \psi^{(n-2)}, \ldots, \psi^{(1)}, u_{x} ; u, \phi^{(1)}, \ldots, \phi^{(n-1)}, \phi^{(n)}\right)}{G W_{n, n}\left(\psi^{(n-1)}, \psi^{(n-2)}, \ldots, \psi^{(1)}, u_{x} ; u, \phi^{(1)}, \ldots, \phi^{(n-2)}, \phi^{(n-1)}\right)}, \\
v^{(n+n)}=\frac{(-1)^{n} G W_{n-1, n}\left(\psi^{(n-2)}, \psi^{(n-3)}, \ldots, \psi^{(1)}, v_{x} ; v, \phi^{(1)}, \ldots, \phi^{(n-2)}, \phi^{(n-1)}\right)}{G W_{n, n}\left(\psi^{(n-1)}, \psi^{(n-2)}, \ldots, \psi^{(1)}, u_{x} ; u, \phi^{(1)}, \ldots, \phi^{(n-2)}, \phi^{(n-1)}\right)},
\end{gathered}
$$

where $\phi^{(j)}=L^{j} u$ and $\left(\phi^{(j)}, \psi^{(j)}\right)$ have the same relation as eq.(159). Also the iteration on the constrained tau functions $\tau_{c}$ of the constrained CMBKP hierarchy as

$$
\tau_{c}^{(n+n)}=G W_{n, n}\left(\psi^{(n-1)}, \psi^{(n-2)}, \ldots, \psi^{(1)}, u_{x} ; u, \phi^{(1)}, \ldots, \phi^{(n-2)}, \phi^{(n-1)}\right) \tau_{c} .
$$

In the above process of calculations, all the elements in above Wronskians must keep being always written in terms of $\Gamma$. In this way, one can keep the new solutions $u^{(n+n)}, v^{(n+n)}$ take values in the algebra $Z_{N}$.

\section{Quantum torus constraint of CMBKP hierar- chy}

In this section, we will focus on the quantum torus symmetry of the CMBKP hierarchy. Firstly we define the operator $\Gamma_{B}$ and the $Z_{N}$-valued Orlov-Shulman's operator $M$ as

$$
\Gamma_{B}=\sum_{i \in \mathbb{Z}_{+}^{\text {odd }}} i t_{i} \partial^{i-1}, \quad M=\Phi \Gamma_{B} \Phi^{-1}
$$

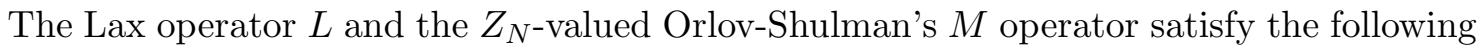
canonical relation

$$
[L, M]=1 .
$$

With the above preparation, it is time to construct additional symmetries for the CMBKP hierarchy in the next part. Then it is easy to get that the operator $M$ satisfy

$$
\begin{aligned}
& {[L, M]=1, M w(z)=\partial_{z} w(z)} \\
& \frac{\partial M}{\partial t_{k}}=\left[\left(L^{k}\right)_{+}, M\right], \quad k \in \mathbb{Z}_{+}^{\text {odd }} .
\end{aligned}
$$


Given any pair of integers $(m, n)$ with $m, n \geq 0$, we will introduce the following $Z_{N^{-}}$ valued operator $B_{m n}$

$$
B_{m n}=M^{m} L^{n}-(-1)^{n} L^{n-1} M^{m} L .
$$

For any $Z_{N}$-valued operator $B_{m n}$ in (80), one has

$$
\frac{\partial B_{m n}}{\partial t_{k}}=\left[\left(L^{k}\right)_{+}, B_{m n}\right], k \in \mathbb{Z}_{+}^{\text {odd }}
$$

Using

$$
\Phi^{*}=\partial \Phi^{-1} \partial^{-1}, \quad \Gamma_{B}^{*}=\Gamma_{B}
$$

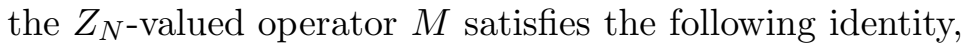

$$
M^{*}=\partial L^{-1} M L \partial^{-1} .
$$

It is easy to check that the $Z_{N}$-valued operator $B_{m n}$ satisfy the B type condition, namely

$$
B_{m n}^{*}=-\partial B_{m n} \partial^{-1} \text {. }
$$

Now we will denote the operator $D_{m n}$ as

$$
D_{m n}:=e^{m M} q^{n L}-L^{-1} q^{-n L} e^{m M} L .
$$

Using eq. (84), the B type property of $D_{m n}$ can be derived as

$$
D_{m n}^{*}=-\partial D_{m n} \partial^{-1} .
$$

Therefore we get the following important B type condition which the $Z_{N}$-valued operator $D_{m n}$ satisfies

$$
D_{m n}^{*}=-\partial D_{m n} \partial^{-1} \text {. }
$$

Then basing on a quantum parameter $q$, the additional flows for the time variable $t_{m, n}, t_{m, n}^{*}$ are defined as follows

$$
\frac{\partial \Phi}{\partial t_{m, n}}=-\left(B_{m n}\right)_{-} \Phi, \quad \frac{\partial \Phi}{\partial t_{m, n}^{*}}=-\left(D_{m n}\right)_{-} \Phi
$$

or equivalently rewritten as

$$
\frac{\partial L}{\partial t_{m, n}}=-\left[\left(B_{m n}\right)_{-}, L\right], \quad \frac{\partial M}{\partial t_{m, n}^{*}}=-\left[\left(D_{m n}\right)_{-}, M\right] .
$$

Generally, one can also derive

$$
\partial_{t_{l, k}^{*}}\left(D_{m n}\right)=\left[-\left(D_{l k}\right)_{-}, D_{m n}\right] .
$$

Using the similar proof as the BKP hierarchy in [14], the additional flows of $\partial_{t_{l, k}^{*}}$ can be proved to be symmetries of the CMBKP hierarchy, i.e. they commute with all $\partial_{t_{n}}$ flows of the CMBKP hierarchy.

The additional flows $\partial_{t_{l, k}}$ of the CMBKP hierarchy form the $W_{\infty}$ algebra similarly as [7] which is about the BKP hierarchy.

Now it is time to identity the algebraic structure of the additional $t_{l, k}^{*}$ flows of the CMBKP hierarchy. 
Theorem 2. The additional flows $\partial_{t_{l, k}^{*}}$ of the CMBKP hierarchy form the positive half of quantum torus algebra, i.e.,

$$
\left[\partial_{t_{n, m}^{*}}, \partial_{t_{l, k}^{*}}\right]=\left(q^{m l}-q^{n k}\right) \partial_{t_{n+l, m+k}^{*}}, \quad n, m, l, k \geq 0 .
$$

Remark: The $t_{l, k}^{*}$ additional flows constitute a nice quantum torus algebra because its basing on a commutative algebra. This is different from the multicomponent BKP whose additional symmetry constitute multi-fold quantum torus algebra [28].

Next, similar to the KP and BKP hierarchy [14, we will consider the quantum torus constraint on the $Z_{N}$-valued tau function of the CMBKP hierarchy.

Similar as [14], one has shown that

$$
\partial_{t_{p, s}} \log w=\left(e^{\tilde{\eta}}-1\right) \frac{\frac{Z_{s}^{(p+1)}}{p+1}(\tau)}{\tau}
$$

where

$$
\tilde{\eta}=\sum_{i \in \mathbb{Z}_{+}^{\text {odd }}} \frac{\lambda^{-i}}{i} \frac{\partial}{\partial t_{i}}
$$

and $Z_{s}^{(p+1)}$ is the generator of the $W_{\infty}^{B}$ algebra. Then with the help of rewriting the quantum torus flow $\partial_{t_{l, k}^{*}}$ in terms of the $\partial_{t_{p, s}}$ flows

$$
\partial_{t_{l, k}^{*}}=\sum_{p, s=0}^{\infty} \frac{l^{p}(k \log q)^{s}}{p ! s !} \partial_{t_{p, s}}
$$

and denoting

$$
L_{l, k}^{B}:=\sum_{p, s=0}^{\infty} \frac{l^{p}(k \log q)^{s}}{p ! s !} \frac{Z_{s}^{(p+1)}}{p+1},
$$

the quantum torus constraint on the $Z_{N}$-valued wave function $w$, i.e.

$$
\partial_{t_{l, k}^{*}} w=0,
$$

will lead to the quantum torus constraint on the $Z_{N}$-valued tau function of the CMBKP hierarchy

$$
L_{l, k}^{B} \tau=c,
$$

where $c$ is a constant.

\section{Conclusions and Discussions}

In this paper, we defined a new multi-component BKP hierarchy which takes values in a commutative subalgebra of $g l(N, \mathbb{C})$. After this, we give the gauge transformation of the commutative multi-component BKP hierarchy. Meanwhile we construct a new constrained CMBKP hierarchy which contains some integrable systems including coupled matrix $K d V$ equations under a certain reduction. After this, the quantum torus symmetry 
and quantum torus constraint of the commutative multi-component BKP hierarchy are constructed. We are looking forward to the possible application of the quantum torus constraint in the topological field theory and enumerate geometry. For the importance of the BKP hierarchy in representation theory and mathematical physics, what is the application of the commutative multi-component BKP hierarchy in other theories such as Frobenius manifold is an interesting question.

Acknowledgements: This work is supported by the National Natural Science Foundation of China under Grant No. 11201251, 11571192, the Zhejiang Provincial Natural Science Foundation under Grant No. LY15A010004, LY12A01007, the Natural Science Foundation of Ningbo under Grant No. 2015A610157 and the K. C. Wong Magna Fund in Ningbo University.

\section{References}

[1] E. Date, M. Kashiwara, M. Jimbo, T. Miwa. Transformation groups for soliton equations, M. Jimbo, T. Miwa, ed. Nonlinear Integrable Systems-Classical and Quantum Theory. Singapore: World Scientific, 1983, 39-119.

[2] M. Toda, Vibration of a chain with nonlinear interaction. J. Phys. Soc. Jpn. $22(1967), 431-436$.

[3] M. Toda, Nonlinear waves and solitons(Kluwer Academic Publishers, Dordrecht, Holland, 1989).

[4] K. Ueno, K. Takasaki, Toda lattice hierarchy, In "Group representations and systems of differential equations" (Tokyo, 1982), 1-95, Adv. Stud. Pure Math., 4, North-Holland, Amsterdam, 1984.

[5] E. Witten, Two-dimensional gravity and intersection theory on moduli space, Surveys in differential geometry, 1(1991), 243-310.

[6] B. A. Dubrovin, Geometry of 2D topological field theories, in Integrable systems and quantum groups (Montecatini Terme, 1993), 120-348, Lecture Notes in Math., 1620, Springer, Berlin, 1996.

[7] M. H. Tu, On the BKP Hierarchy: Additional Symmetries, Fay Identity and Adler-Shiota-van Moerbeke Formula, Lett. Math. Phys. 81(2007),93-105.

[8] J. S. He, Y. Cheng and R. A. Römer, Solving bi-directional soliton equations in the KP hierarchy by gauge transformation, JHEP 03(2006), 103.

[9] J. S. He, Z. W. W, Y. Cheng. Gauge transformations for the constrained CKP and BKP hierarchies, J. Math. Phys., 48(2007), 113519.

[10] C. Z. Li, J. S. He, Block algebra in two-component BKP and D type DrinfeldSokolov hierarchies, J. Math. Phys. 54, 113501(2013).

[11] C. Z. Li, J. S. He, Y. C. Su, Block type symmetry of bigraded Toda hierarchy, J. Math. Phys. 53(2012), 013517.

[12] C. Z. Li, J. S. He, Dispersionless bigraded Toda hierarchy and its additional symmetry, Reviews in Mathematical Physics, 24(2012), 1230003.

[13] C. Z. Li, J. S. He, Y. C. Su, Block (or Hamiltonian) Lie symmetry of dispersionless D type Drinfeld-Sokolov hierarchy, Commun. Theor. Phys. 61(2014), 431-435. 
[14] C. Z. Li, J. S. He, Quantum Torus symmetry of the KP, KdV and BKP hierarchies, Lett. Math. Phys. 104(2014), 1407-1423.

[15] C. Z. Li, J. S. He, Supersymmetric BKP systems and their symmetries, Nuclear Physics B 896(2015), 716-737.

[16] V. G. Kac and J. W. van de Leur, The n-component KP hierarchy and representation theory, J. Math. Phys. 44 (2003) 3245.

[17] M. Adler, P. van Moerbeke and P. Vanhaecke, Moment matrices and multicomponent KP, with applications to random matrix theory, Commun. Math. Phys. 286(2009), 1.

[18] L. V. Bogdanov, B. G. Konopelchenko. Analytic-bilinear approach to integrable hierarchies. II. Multicomponent KP and 2D Toda lattice equations. J. Math. Phys. 39:9 (1998) 4701-4728.

[19] V. G. Kac, J. W. van de Leur. The geometry of spinors and the multicomponent BKP and DKP hierarchies. CRM Proc. Lect. Notes 14 (1998) 159-202.

[20] M. Mañas, Luis Martínez Alonso. From Ramond fermions to Lame equations for orthogonal curvilinear coordinates. Phys. Lett. B 436:3 4 (1998) 316-322.

[21] C. Álvarez Fernández, U. Fidalgo Prieto, M. Mañas, The multicomponent 2D Toda hierarchy: generalized matrix orthogonal polynomials, multiple orthogonal polynomials and Riemann-Hilbert problems, Inverse Problems, 26(2010), 055009.

[22] C. Álvarez Fernández, U. Fidalgo Prieto, and M. Mañas, Multiple orthogonal polynomials of mixed type: Gauss-Borel factorization and the multi-component 2D Toda hierarchy, Advances in Mathematics, 227(2011), 1451-1525.

[23] C. Z. Li, J. S. He, On the extended multi-component Toda hierarchy, Math. Phys. Analyis and Geometry 17(2014), 377-407.

[24] D. F. Zuo, Local matrix generalizations of $W$-algebras, arXiv:1401.2216.

[25] C. Z. Li, J. S. He, The extended $Z_{N}$-Toda hierarchy, Theoretical and Mathematical Physics, 185(2015), 1614-1635.

[26] J. S. He, L. Zhang, Y. Cheng and Y. S. Li, Determinant representation of Darboux transformation for the AKNS system, Sci. China A, 12(2006), 1867-78.

[27] C. Z. Li, J. S. He, and K. Porsezian, Rogue waves of the Hirota and the MaxwellBloch equation, Physical Review E 87(2013), 012913.

[28] C. Z. Li, Dispersionless multicomponent BKP Hierarchy and perturbative quantum torus symmetries, submitted. 